\title{
ON MINIMAL IRREGULAR $p$-GROUPS
}

\author{
Dedicated to the memory of Hanna Neumann
}

\section{J. R. J. GROVES}

(Received 5 May 1972)

Communicated by M. F. Newman

\section{Introduction}

The study of minimal irregular $p$-groups was initiated by Mann [9]. He defines such a group to be a finite irregular $p$-group in which every proper section is regular. He then deduces a large number of properties of such groups and shows, by construction, that there is no bound on their exponent. In this note methods involving varieties of groups are used to show that all minimal irregular $p$-groups can be 'built up' from cyclic groups, groups of exponent $p$ and a relatively small class of metabelian minimal irregular $p$-groups. To phrase this more precisely, say that a group $P$ is derivable from a family of groups $\left\{Q_{i}\right\}$ if $P$ is a homomorphic image of a subdirect product of the $Q_{i}$. Then we will prive as Theorem 2.2:

a minimal irregular p-group of exponent $p^{n}$ and nilpotency class $c$ is derivable from a family of three groups:

i) a cyclic group of order $p^{n}$,

ii) a 2-generator monolithic group of exponent $p$ and class $c$,

iii) a minimal irregular p-group whi $h$ is metabelian, has class $p$ and has exponent $p^{2}$.

The key to proving this theorem is the observation that Mann's results imply that every minimal irregular $p$-group is a 2-generator group and satisfies the law

$$
\left(x_{1}\left[x_{2}, x_{3}\right]\right)^{p}=x_{1}^{p} \text {. }
$$

Denote the variety generated by all 2-generator groups which have this law by $\mathfrak{D}_{p}$. Also, denote the variety of all abelian groups by $\mathfrak{U}$, the variety generated by all 2-generator groups of exponent $p$ by $\mathfrak{B}_{p}$ and the variety generated by the wreath product of two cyclic groups of order $p$ by $\mathfrak{D}_{p}^{*}$. Then the major step in the proof of Theorem 2.2 is to show that $\mathfrak{D}_{p}$ is the join of $\mathfrak{A}$ and $\mathfrak{B}_{p}^{\prime}$ and $\mathfrak{D}_{p}^{*}$ (this appears as Lemma 2.1). Weichsel [13] has studied a restricted type of minimal 
irregular $p$-group-which he calls a just irregular $p$-group-defined by the additional property that all proper subvarieties of the variety it generates are regular (a variety of $p$-groups being regular if each finite group of the variety is regular). Call such a variety minimal $p$-irregular. Clearly $\mathfrak{D}_{p}^{*}$ is minimal $p$-irregular (see, for example, Kovács and Newman [8], where $\mathfrak{D}_{p}^{*}$ is denoted by $\mathfrak{I}(p, p)$ ). The main theorem of Section 3 (Theorem 3.6) gives classes of varieties which have no other minimal $p$-irregular subvariety.

Let $\mathfrak{U}$ be an irregular variety of p-groups. Then $\mathfrak{D}_{p}^{*}$ is the only minimal p-irregular subvariety of $\mathfrak{U}$ if $\mathfrak{U}$ is a subvariety of $\mathfrak{N}_{3 p-3}$ or if $\mathfrak{U}$ is a subvariety of $\mathfrak{N}_{2} \mathfrak{A}$ or if $p=2,3$ or 5 .

The proof of this result and the further question of the necessity of the conditions placed on $\mathfrak{U}$ appear to depend on the structure of the free group of exponent $p$ and rank 2. This statement is made more precise in Proposition 3.3 where a necessary and sufficient condition for the existence of other minimal $p$-irregular varieties is obtained in terms of the existence of a certain, well-defined, type of section of this free group. Finally, we use Theorem 3.6 to give criteria for a variety satisfying one or more of the conditions of the theorem to be regular.

For unexplained notation, terminology and basic results, we refer to Hanna Neumann's book [11]. We will, however, differ from the notation used there in a number of respects; we use $\mathfrak{B}(G)$ to denote the verbal subgroup of the group $G$ corresponding to the variety $\mathfrak{B}$ and we do not reserve $G, H$ for relatively free groups nor $F$ for an absolutely free group. If $\mathfrak{U}$ is a subvariety of $\mathfrak{Z}$ we write $\mathfrak{U} \leqq \mathfrak{B}$. If $H$ is a subgroup of a group $G$ and $K$ is a normal subgroup of $H$, then $H / K$ is a section of $G$. It is proper unless $K=\{1\}$ and $H=G$.

Throughout this note, $p$ will denote a fixed prime number. A finite $p$-group $P$ is said to be regular if, whenever $g, h \in P$, there exist elements $d_{1}, \cdots, d_{s}$ of the derived group of the group generated by $g$ and $h$ such that $(g h)^{p}=g^{p} h^{p} d_{1}^{p} \cdots d_{s}^{p}$. A variety of $p$-groups is regular if each finite group of the variety is regular.

If $r$ is a natural number, we will write $[g, r h]$ for the commutator $[g, h, \cdots, h]$ with $h$ repeated $r$ times. If a group $P$ satisfies the law $\left[x_{1}, r x_{2}\right]=1$, we say that $P$ has the $r^{\prime}$ th Engel condition. If, for all elements $g$ and $h$ of $P,[g, r h] \in \mathfrak{N}_{r+1}$ $(g p(g, h))$, then we say that $G$ has the $r$ 'th Engel congruence. A variety of groups has the $r$ 'th Engel condition (congruence) if each group of the variety does so.

I am indebted to Dr. M. F. Newman for a suggestion which led to the consideration of the topics discussed here and for his constructive criticism of the initial presentation.

\section{Minimal irregular $p$-groups}

It follows immediately from results of Mann $[9$; Theorem 2, a) and $k$ )] that a minimal irregular $p$-group is a 2-generator group which satisfies the law

$$
\left(x_{1}\left[x_{2}, x_{3}\right]\right)^{p}=x_{1}^{p}
$$


The first half of this section will be devoted to studying the structure of the variety $\mathfrak{D}_{p}$ generated by all 2-generator groups satisfying this law. To begin with, let us extract a few consequences of the law. If $P$ is a group of $\mathfrak{D}_{p}$, then $P^{\prime}$ has exponent $p$ and $p$ 'th powers of elements of $P$ are central in $P$; the former is immediate and the latter can be seen by replacing $x_{2}$ by $x_{1}$ in the law. If $P$ is also regular, then it is $p$-abelian-that is, it satisfies the law $\left(x_{1} x_{2}\right)^{p}=x_{1}^{p} x_{2}^{p}$.

Let $\mathfrak{D}_{p}^{*}$ denote the subvariety of $\mathfrak{D}_{p}$ generated by the wreath product of two cyclic groups of order $p$. This variety plays a central role throughout this note and the next step in the argument will be to describe some facets of the structure of its free group of rank 2. Denote this group by $G$ and let $\{x, y\}$ be a free generating set of $G$. The description of the subvariety lattice of $\mathfrak{U}_{p} \mathfrak{A}_{p}$ in Kovács and Newman $\left[8 ;\right.$ p. 133] shows that $\mathfrak{D}_{p}^{*}$ has a unique maximal subvariety $\mathfrak{N}=\left(\mathfrak{D}_{p}^{*} \wedge \mathfrak{B}_{p}\right) \vee \mathfrak{A}_{p^{2}}$. By a result of Meier-Wunderli [10], 2-generator metabelian groups of exponent $p$ have class at most $p-1$ and so $\mathfrak{N}(G)=\mathfrak{R}_{p-1}(G)$ $=N$ say. As $G$ has class precisely $p, N$ is not trivial and so is the unique minimal verbal subgroup of $G$. Further, it follows from 4.05 and 4.06 of [8], that $N$ has a free generating set $\{[x, r y,(p-1-r) x] \mid 1 \leqq r \leqq p-1\}$ and so that $N$ is an elementary abelian group of order $p^{p-1}$.

We will exhibit another basis for $N$. Let $B$ denote $\mathfrak{B}_{p}(G)$. As we remarked above, 2-generator metabelian groups of exponent $p$ have class at most $p-1$ and so $B \geqq N$. Also, as a consequence of the law defining $\mathcal{D}_{p}$, if $g \in G$ and $h \in G^{\prime}$, then $(g h)^{p}=g^{p}$. Hence the obvious generating set for $B$, consisting of all $p^{\prime}$ th powers of elements of $G$, may be refined to the set $\left\{x^{p}, y^{p},\left(x y^{r}\right)^{p} \mid 1 \leqq r \leqq p-1\right\}$. Let $w_{r}$ denote $\left(x y^{r}\right)^{p} y^{-r p} x^{-p}$; then $\left\{x^{p}, y^{p}, w_{r} \mid 1 \leqq r \leqq p-1\right\}$ also generates $B$. As $F / N$ is regular, but not of exponent $p, B / N=\mathfrak{B}_{p}(F / N)$ is freely generated by $x^{p} N$ and $y^{p} N$ and the $w_{r}$ belong to $N$. Thus, $\left\{w_{r} \mid 1 \leqq r \leqq p-1\right\}$ generates $N$ and so, as $N$ is elementary abelian of order $p^{p-1}$, this is a basis of $N$.

We are now ready to prove the following lemma describing the structure of $\mathfrak{D}_{p}$. The variety generated by all 2-generator groups of exponent $p$ is denoted by $\mathfrak{B}_{p}^{\prime}$.

\section{LEMMA 2.1. $\mathfrak{D}_{p}=\mathfrak{A} \vee \mathfrak{B}_{p}^{\prime} \vee \mathfrak{D}_{p}^{*}$}

Proof. Clearly $\mathfrak{A}$ and $\mathfrak{B}_{p}^{\prime}$ and $\mathfrak{D}_{p}^{*}$ are subvarieties of $\mathfrak{D}_{p}$. Let $F$ denote $F_{2}\left(\mathfrak{D}_{p}\right)$ and let $D, B_{1}$ denote $\mathfrak{D}_{p}^{*}(F)$ and $\mathfrak{B}_{p}(F)$ respectively. The set $\{x, y\}$ will denote a free generating set of $F$ and the elements $w_{r}$ are defined similarly. As with our previous remarks, $B_{1}$ is a central subgroup of $F$ generated by $x^{p}, y^{p}$ and the $w_{r}$. Let $T$ denote the subgroup of $B_{1}$ generated by the $w_{r}$.

Since the elements $w_{r} D$ are independent, $T \cap D=\{1\}$. Let $g \in F^{\prime} \cap B_{1}$. Then $g T=x^{p k} y^{p l} T$ for some integers $k$ and $l$. Thus, as $T \leqq F^{\prime}, x^{p k} y^{p l} \in F^{\prime}$ and so, because $x$ and $y$ are free generators of $F, x^{p k}=y^{p l}=1$. Hence $F^{\prime} \cap B_{1} \leqq T$ and so $F^{\prime} \cap B_{1} \cap D=\{1\}$. It follows that 


$$
\operatorname{var}(F)=\operatorname{var}\left(F / F^{\prime}\right) \vee \operatorname{var}\left(F / B_{1}\right) \vee \operatorname{var}(F / D),
$$

which is what we set out to prove.

The groundwork is laid for the description of minimal irregular p-groups. It follows immediately from the previous lemma that such a group is derivable from a set of three groups, one each from $\mathfrak{U}, \mathfrak{B}_{p}^{\prime}$ and $\mathfrak{D}_{p}^{*}$. We can, however, improve on this.

THEOREM 2.2 A minimal irregular p-group of exponent $p^{n}$ and nilpotency class $c$ is derivable from a family of three groups:

i) a cyclic group of order $p^{n}$,

ii) a 2-generator monolithic group of exponent $p$ and class $c$,

iii) a minimal irregular p-group which is metabelian, has class $p$ and has exponent $p^{2}$.

Proof. Let $P$ be a minimal irregular $p$-group of exponent $p^{n}$ and class $c$. Then

$$
P \in \mathfrak{B}_{p^{n}} \wedge \mathfrak{N}_{c} \wedge \mathfrak{D}_{p}
$$

clearly $n \geqq 2$ and $c \geqq p$. Hence, by Lemma 2.1 and two applications of the modular law,

$$
P \in \mathfrak{N}_{p^{n}} \vee\left(\mathfrak{B}_{p}^{\prime} \wedge \mathfrak{N}_{c}\right) \vee \mathfrak{D}_{p}^{*}
$$

Hence $P$ is a homomorphic image of the free group of rank 2, $H$ say, of this join variety. By 15.82 of Hanna Neumann [11], $H$ is a subdirect product of the free groups of rank 2 of the varieties $\mathfrak{A}_{p^{n}}, \mathfrak{B}_{p}^{\prime} \wedge \mathfrak{N}_{c}$ and $\mathfrak{D}_{p}^{*}$; denote these groups by $A_{1}, B_{1}$ and $C_{1}$ respectively.

We have shown that $P$ is derivable from $A_{1}, B_{1}$ and $C_{1}$. Choose homomorphic images $A_{2}, B_{2}$ and $C_{2}$ so that $P$ is derivable from these groups but $P$ is not derivable from the set obtained by replacing any one of them by a homomorphic image. Then $A_{2}, B_{2}$ and $C_{2}$ are still 2-generator groups. Since all the proper sections of $P$ are regular, they satisfy the law $\left(x_{1} x_{2}\right)^{p}=x_{1}^{p} x_{2}^{p}$; since $P$ is irregular, it does not. Hence $P$ is a critical group and so, in particular, monolithic. It now follows, by 3.6 of Kovács and Newman [7], that $A_{2}, B_{2}$ and $C_{2}$ are also monolithic (note that the present construction is slightly different from that of [7]; the proofs, however, are equally valid).

We have shown, so far, that $A_{2}$ is a cyclic group and that $B_{2}$ is a 2-generator monolithic group of exponent $p$. To complete the descriptions given in i) and ii) of the statement of the theorem, observe that the exponent of $A_{2}$ and the class of $B_{2}$ are determined by the exponent and class, respectively, of $P$. It remains to show that $C_{2}$ satisfies iii). As $C_{2} \in \mathfrak{D}_{p}^{*}$, it will suffice to show that it is minimal irregular.

It is clear that $C_{2}$ is irregular. By 1.3 of [7], and the fact that $C_{2}$ is monolithic, every proper homomorphic image of $C_{2}$ lies in a proper subvariety of $\mathfrak{D}_{p}^{*}$. How- 
ever, as we have already remarked, every proper subvariety of $\mathfrak{D}_{p}^{*}$ is regular; therefore every proper homomorphic image of $C_{2}$ is regular. Since $C_{2}$ is a 2generator group, it is a homomorphic image of $F_{2}\left(D_{p}^{*}\right)$. But it is easily verified that every proper subgroup of the latter group is regular (in fact of class $p-1$ ). Hence every proper subgroup of $C_{2}$ is regular and so $C_{2}$ is a minimal irregular $p$-group. The proof of the theorem is complete.

Since $p$-groups of class $p-1$ are regular, a minimal irregular $p$-group has class at least $p$. Also, the positive solution of the Restricted Burnside Problem for groups of exponent $p$, due to Kostrikin [6], shows that there is a bound- $c(p)$ say-on the class of finite 2-generator groups of exponent $p$. Hence, by Theorem 2.2 , the class of a minimal irregular $p$-group cannot exceed the maximum of $p$ and $c(p)$ (this maximum is clearly $c(p)$ unless $p=2$ ). Avinoam Mann (private communication) has constructed minimal irregular $p$-groups of every possible class between these two bounds. This situation is considerably different to the situation for minimal $p$-irregular varieties, as we shall see in the next section.

\section{Minimal $p$-irregular varieties}

We begin this section by noting a varietal consequence of Lemma 2.1 .

LEMMA 3.1. Let $\mathfrak{B}$ be a minimal p-irregular variety. Then

$$
\mathfrak{B} \leqq \mathfrak{D}_{p}^{*} \vee \mathfrak{B}_{p}^{\prime} \text {, and }
$$

ii)

$$
\mathfrak{D}_{p}^{*} \leqq \mathfrak{B} \vee \mathfrak{B}_{p}^{\prime} \text {. }
$$

PRoOF. It follows immediately from the proof of Lemma 2.1 that $F_{2}\left(\mathfrak{D}_{p}\right)$ has a minimal verbal subgroup corresponding to the variety $\mathfrak{U} \vee \mathfrak{B}_{p}^{\prime}$ (the subgroup $T$ in the notation of 2.1). But $\mathfrak{A} \vee \mathfrak{B}_{p}^{\prime}$ is clearly regular and so, if $\mathfrak{U}$ is an irregular subvariety of $\mathfrak{D}_{p}$ which can be generated by its 2-generator groups, $\mathfrak{U} \vee \mathfrak{A} \vee \mathfrak{B}$; $=\mathfrak{D}_{p}$. Thus, if $\mathfrak{U}$ has exponent $p^{2}$, then by an application of the modular law, $\mathfrak{D}_{p} \wedge \mathfrak{B}_{p^{2}}=\mathfrak{U} \vee \mathfrak{A}_{p^{2}} \vee \mathfrak{B}_{p}^{\prime}=\mathfrak{U} \vee \mathfrak{B}_{p}^{\prime}$. The lemma follows after observing that a minimal $p$-irregular variety has exponent $p^{2}$, by a theorem of Weichsel [13], and can be generated by its 2 -generator groups.

The next lemma may be deduced from Theorem 1.4 of Weichsel [12]. The previous lemma, however, enables us to give a brief and independent proof.

Lemma 3.2 (Weichsel). The only metabelian minimal p-irregular variety is $\mathfrak{D}_{p}^{*}$.

ProOF. If $\mathfrak{B}$ is a metabelian minimal $p$-irregular variety, then

$$
\mathfrak{B} \leqq\left(\mathfrak{D}_{p}^{*} \vee \mathfrak{B}_{p}^{\prime}\right) \wedge \mathfrak{A}^{2}=\mathfrak{D}_{p}^{*} \wedge\left(\mathfrak{B}_{p}^{\prime} \vee \mathfrak{U}^{2}\right)
$$

by Lemma 3.1 and an application of the modular law. But $\mathfrak{B}_{p}^{\prime} \wedge \mathfrak{U}^{2} \leqq \mathfrak{D}_{p}^{*}$ (see, 
for example, the description of the lattice of subvarieties of $\mathfrak{A}_{p} \mathfrak{A}_{p}$ in [8; p. 133]). Thus $\mathfrak{B}=\mathfrak{D}_{p}^{*}$.

It was stated in the introduction that the existence of minimal $p$-irregular varieties other than $\mathfrak{D}_{p}^{*}$ apparently depends on the structure of $F_{2}\left(\mathfrak{B}_{p}\right)$. We must justify this statement. In doing so, we will obtain a criterion for the existence of such varieties which will enable us to extend the class of varieties for which we can show that $\mathfrak{D}_{p}^{*}$ is the only minimal $p$-irregular subvariety.

Suppose that $\mathfrak{B}$ is a minimal $p$-irregular variety and that $\mathfrak{B} \neq \mathfrak{D}_{p}^{*}$. Let $F=F_{2}\left(D_{p}\right)$ and let $D, V$ and $N$ denote $\mathfrak{D}_{p}^{*}(F), \mathfrak{B}(F)$ and $D \mathfrak{N}_{p-1}(F)$. If $c$ is a natural number, denote $\left(\mathfrak{B}_{p}^{\prime} \wedge \mathfrak{N}_{c}\right)(F)$ by $B_{c}$. Finally, denote the semigroup of endomorphisms of $F$ by $\Omega$ and consider $F$ as an $\Omega$-group in the natural way. The subgroups defined above are fully invariant subgroups of $F$ and therefore $\Omega$ subgroups.

Suppose that $\mathfrak{B}$ has class $c$; clearly $c \geqq p$. By Lemma 3.1 and the modular law,

$$
\mathfrak{B} \leqq \mathfrak{D}_{p}^{*} \vee\left(\mathfrak{B}_{p}^{\prime} \wedge \mathfrak{N}_{c}\right) \text { and } \mathfrak{D}_{p}^{*} \leqq \mathfrak{B} \vee\left(\mathfrak{B}_{p}^{\prime} \wedge \mathfrak{N}_{c}\right)
$$

or, in terms of $\Omega$-subgroups of $F$,

$$
V \geqq D \cap B_{c} \text { and } D \geqq V \cap B_{c} \text {. }
$$

Hence, $V \cap D \geqq V \cap B_{c}=D \cap B_{c}$. Now consider the following pair of $\Omega$ isomorphisms:

$$
V D / D \cong V / V \cap D \text { and } V / V \cap B_{c} \cong V B_{e} / B_{c} .
$$

As $V \cap D \geqq V \cap B_{c}$, these isomorphisms imply that $V B_{c} / B_{c}$ has an $\Omega$-section isomorphic to $V D / D$. Since $\mathfrak{B} \neq \mathfrak{D}_{p}^{*}$, however, $V \leqq D$ and so $V D / D \geqq N / D$ the unique minimal normal subgroup of $F / D$. Hence $F / B_{c}$ has an $\Omega$-section which is $\Omega$-isomorphic to $N / D$.

For use later on, we elicit some extra information. Since $B_{c} D / D$ is not trivial and

$$
B_{c} D / D \cong B_{c} / D \cap B_{c}=B_{c} / V \cap B_{c} \cong B_{c} V / V,
$$

it follows that $F / V$ has an $\Omega$-subgroup- $V_{1} / V$ say-which is also isomorphic to $N / D$. Clearly $V_{1} / V$ is the unique minimal verbal subgroup of $F / V$.

It is not difficult to check - and we omit the details-that the factor semigroup of $\Omega$ by the kernel of its representation on $N / D$ is the group $G L(2, p)$ of all nonsingular transformations of a 2-dimensional space over the field with $p$ elements. Thus $N / D$ provides an irreducible representation of $G L(2, p)$ and what has been shown above amounts to the statement that $F / B_{c}$ and $F / V$ have $\Omega$-sections which provide representations of $G L(2, p)$ equivalent to this one. (This is a simple example of a general process, due to Higman [3], for studying varieties by means of representations of general linear groups.) 
We can now state the following proposition, which includes what has been proved so far.

Propositron 3.3. The following statements are equivalent:

i) there exists a minimal p-irregular variety which is different from $\mathfrak{D}_{p}^{*}$ and of class at most $c$,

ii) the free group of rank 2 of $\mathfrak{N}_{c} \wedge \mathfrak{B}_{p}$ has an $\Omega$-chief section which provides a representation of $G L(2, p)$ equivalent to that provided by $N / D$.

Proof. We have already shown that i) implies ii) and so it remains to prove the converse. Suppose that ii) holds. Then, retaining the previous notation, there is an $\Omega$-section $S / T$, of $F / B_{c}$, which is $\Omega$-isomorphic to $N / D$. To prove the proposition, we will construct a verbal ( $\Omega$-) subgroup $W$ of $F$ satisfying:

i) $T \cap D \leqq W \leqq(T \cap N)(S \cap D)$,

ii) $W \nsubseteq D$,

iii) $F / W$ is irregular.

If $W$ satisfies these conditions, then $\operatorname{var}(F / W)$ is irregular and so must contain a minimal $p$-irregular variety. As $W \geqq D$, this cannot be $\mathfrak{D}_{p}^{*}$. Since $W \geqq T \cap D$ $\geqq B_{c} \cap D \geqq \mathfrak{N}_{c}(F), \operatorname{var}(F / W)$ has class at most $c$. Hence $\operatorname{var}(F / W)$ contains a minimal $p$-irregular variety which is different from $\mathfrak{D}_{p}^{*}$ and of class at most $c$.

The first step in constructing $W$ is to show that both $T \cap N / T \cap D$ and $S \cap D / T \cap D$ are non-trivial. Suppose that $T \cap N=T \cap D$. Then $D \geqq N \cap T$; but $F / N$ and $F / T$ are both regular groups of $\mathfrak{D}_{p}$ and so satisfy the law $\left(x_{1} x_{2}\right)^{p}$ $=x_{1}^{p} x_{2}^{p}$. Hence $F / D$ also satisfies this law and so $\mathfrak{D}_{p}^{*}$ is regular-a contradiction. Now suppose that $S \cap D=T \cap D$. Then $T \geqq S \cap D$ and so $T=(S \cap D) T$ $=S \cap T D$, by the modular law. Hence,

$$
S D / T D=S(T D) / T D \cong S / S \cap T D=S / T \cong N / D .
$$

As $T D \geqq N$, this implies that $F / N$ has an $\Omega$-section isomorphic to $N / D$. But it can easily be checked that the order of an $\Omega$-chief section of $F / N$ can be at most $p^{p-2}$-another contradiction.

We have shown that $T \cap N / T \cap D$ and $S \cap D / T \cap D$ are non-trivial $\Omega$-sections of $F$. But,

$$
T \cap N / T \cap D=T \cap N /(T \cap N) \cap D \cong(T \cap N) D / D \leqq N / D .
$$

Hence, as $N / D$ is an $\Omega$-chief section and $T \cap N / T \cap D$ is non-trivial, $N / D$ $\cong T \cap N / T \cap D$. Similarly $S / T \cong S \cap D / T \cap D$. But $S / T$ and $N / D$ are $\Omega$-isomorphic. Thus, $T \cap N / T \cap D$ and $S \cap D / T \cap D$ are $\Omega$-isomorphic; let $\sigma: T \cap N / T \cap D \rightarrow S \cap D / T \cap D$ be an $\Omega$-isomorphism. Let $W$ denote

$$
\left\{g^{-1} g \sigma \mid g \in T \cap N / T \cap D\right\} \text {. }
$$


As $\sigma$ is an $\Omega$-isomorphism, $\bar{W}$ is an $\Omega$-subgroup of $F / T \cap D$; let $W$ be the corresponding $\Omega$-subgroup of $F$. Clearly $W$ satisfies $T \cap D \leqq W \leqq(T \cap N)(S \cap D)$. Also, $W \cap T=W \cap D=T \cap D$ and so $W \$ D$. Finally, because $D \geqq W \cap T$, and $F / D$ is irregular, it follows that $F / W$ is irregular. Hence $W$ satisfies the requirements demanded of it and so the proof of the proposition is complete.

Before this proposition is used to deduce results on the class of minimal $p$-irregular varieties, we state a lemma to justify some of the manipulations that will occur with commutators. It is simply Corollary 1.1 of Brisley [1] tailored to meet our requirements.

LEMMA 3.4 Let $H$ be a relatively free p-group which is nilpotent of class $c$ and suppose that $\{x, y\}$ is a free generating set of $H$. Suppose, also, that $\prod_{i=1}^{p-1} u_{i}=1$, where each $u_{i}$ is a product of commutators, in $x$ and $y$, each of weight $c$ and order dividing $p$, and each involving, for some $m \geqq 0, i+m(p-1)$ occurrences of $y$. Then $u_{i}=1(1 \leqq i \leqq p-1)$.

The first half of the next result is due to Weichsel [13]; it is included here as its proof appears naturally on the way to proving the second half.

Proposition 3.5. A minimal p-irregular variety $\mathfrak{B}$ has class $k(p-1)+1$ for some $k \geqq 1$. If $\mathfrak{B} \neq \mathfrak{D}_{p}^{*}$, then $k \geqq 3$.

Proof. The proposition is immediate if $\mathfrak{V}=\mathfrak{D}_{p}^{*}$; suppose, therefore, that this is not the case. Let $c$ be the class of $\mathfrak{B}$ and define $F, N, D, V$ and $B_{c}$ as in the proof of Proposition 3.3. We begin the proof by showing how the proposition follows from:

(1) let $S / T$ be an $\Omega$-section of $F$ which is $\Omega$-isomorphic to $N / D$ and suppose that $F / T$ is nilpotent of class $d$ and that $S / T \leqq \mathfrak{N}_{d-1}(F / T)$; then,

a) $d=k(p-1)+1$ for some $k \geqq 1$,

b) if $F / T$ has the $(p-1)$ 'th Engel congruence, $k \geqq 3$.

Firstly, as we remarked in the proof of Proposition 3.3, $F / V$ has a unique minimal $\Omega$-subgroup $V_{1} / V$ which is $\Omega$-isomorphic to $N / D$; the uniqueness implies that $V_{1} / V \leqq \mathfrak{N}_{c-1}(F / V)$. Thus part a) of (1) implies that $c=k(p-1)+1$ for some $k \geqq 1$, which proves the first half of the theorem.

Secondly, the (supposed) existence of $\mathfrak{B}$ guarantees, by Proposition 3.3 , the existence of an $\Omega$-section of $F / B_{c}$ which is $\Omega$-isomorphic to $N / D$. Choose such a section $S / T$ which is maximal in the sense that no section of $F / S$ is $\Omega$-isomorphic to $N / D$. Let $b$ be the class of $F / T$. The maximality of $S / T$ implies that $S / T$ $\leqq \mathfrak{N}_{b-1}(F / T)$. Also, $F / T$ is a group of exponent $p$ and so satisfies the $(p-1)$ 'th Engel congruence (see, for example, Huppert [4; III, 9.7]). Hence, by (1), $b \geqq 3(p-1)+1$. But, clearly, $c \geqq b$ and the second half of the theorem follows. 
It remains to prove statement (1). Let $\{x, y\}$ be a free generating set of $F$ and abbreviate $x T, y T$ by $\bar{x}, \bar{y}$ and $x D, y D$ by $x^{*}, y^{*}$. Let $\sigma$ be an $\Omega$-isomorphism from $N / D$ to $S / T$ and denote $\left[x^{*}, r y^{*},(p-1-r) x^{*}\right]$ by $z_{r}$ and $z_{r} \sigma$ by $v_{r}(1 \leqq r \leqq p-1)$. Observe that $\left\{z_{r} \mid 1 \leqq r \leqq p-1\right\}$ is a basis of $N / D$ and consequently $\left\{v_{r} \mid 1 \leqq r\right.$ $\leqq p-1\}$ is a basis of $S / T$. Throughout this proof, $\lambda$ will denote a fixed primitive root of $p$.

Let $\phi \in \Omega$ be defined by

$$
\phi: x \mapsto x^{\lambda}, \quad y \mapsto y^{\lambda} .
$$

(Here, as with other endomorphisms of $F$, we will also use $\phi$ to denote the endomorphism induced modulo an $\Omega$-subgroup of $F$.) Then,

$$
z_{r} \phi=z_{r}^{\lambda p}=z_{r}^{\lambda} \quad\left(\text { as } \lambda^{p-1} \equiv 1 \bmod p\right) .
$$

Hence,

$$
v_{r} \phi=\left(z_{r} \sigma\right) \phi=\left(z_{r} \phi\right) \sigma=\left(z_{r}^{\lambda}\right) \sigma=v_{r}^{\lambda}
$$

But $S / T \leqq U_{d-1}(F / T)$ and so each element of $S / T$, in particular each $v_{r}$, can be written as a product of commutators, in $\bar{x}$ and $\bar{y}$, of weight $d$. Hence $v_{r} \phi=v_{r}^{\lambda^{d}}$. Thus $v_{r}^{\lambda^{d}}=v_{r}^{\lambda}$ and so, as $v_{r}$ has order $p$, it follows that $p \mid\left(\lambda^{d}-\lambda\right)$. Therefore $d=k(p-1)+1$ for some $k$; clearly $k \geqq 1$. This completes the proof of part a).

Write $v_{r}$ in the form

$$
v_{r}=\prod_{i=1}^{p-1} v_{r}(i)
$$

where each $v_{r}(i)$ is a product of commutators, in $\bar{x}$ and $\bar{y}$, each of weight $d$ and each involving, for some $m \geqq 0, i+m(p-1)$ occurrences of $\bar{y}$. Let $\rho \in \Omega$ be defined by

$$
\rho: x \mapsto x, \quad y \mapsto y^{\lambda} .
$$

Then $v_{r}(i) \rho=v_{r}(i)^{2 i}$ and so

But,

$$
v_{r} \rho=\prod_{i=1}^{p-1} v_{r}(i)^{\lambda !}
$$

Hence,

$$
v_{r} \rho=\left(z_{r} \sigma\right) \rho=\left(z_{r} \rho\right) \sigma=\left(z_{r}^{\lambda r}\right) \sigma=v_{r}^{\lambda r} .
$$

$$
\prod_{i=1}^{p-1} v_{r}(i)^{\lambda i}=\prod_{i=1}^{p-1} v_{r}^{\lambda r}
$$

It follows, by Lemma 3.4, that $v_{r}(i)^{\lambda^{i}-\lambda^{r}}=1$. Therefore $v_{r}(i)=1$ unless $i=r$ and so $v_{r}$ is a product of commutators each involving, for some $m \geqq 0, r+m(p-1)$ occurrences of $\bar{y}$. 
Since $F / T$ has the $(p-1)$ 'th Engel congruence, a commutator involving $k(p-1)$ occurrences of $\bar{y}$-and so only one occurrence of $\vec{x}$-is trivial. In particular, this implies that $k>1$. For if $k=1, v_{p-1}$ involves $p-1$ occurrences of $\bar{y}$ and so is trivial-which is in contradiction to the fact that $\left\{v_{r}\right\}$ is a basis of $S / T$.

Suppose that $k=2$ and let $\tau \in \Omega$ be defined by

$$
\tau: x \mapsto x, \quad y \mapsto x y .
$$

Because commutators involving $2(p-1)$ occurrences of $\bar{y}$ are trivial, $v_{p-1}$ is a product of commutators involving precisely $p-1$ occurrences of $\bar{y}$ and so,

$$
v_{p-1} \tau=\prod_{i=1}^{p-1} u_{i}
$$

where $u_{i}$ is a product of commutators involving $i$ (but not $i+(p-1)$ ) occurrences of $\bar{y}$. It is easily verified, however, that

$$
z_{p-1} \tau=\prod_{i=1}^{p-1} z_{i}^{n(i)}
$$

where each $n(i)$ is an integer prime to $p$. So, as usual,

$$
\prod_{i=1}^{p-1} u_{i}=\prod_{i=1}^{p-1} v_{i}^{n(i)}
$$

Hence, by Lemma 3.4, $u_{1}=v_{1}^{n(1)}$. But $u_{1}$, being a product of commutators involving only one occurrence of $\bar{y}$, is trivial. Thus $v_{1}=1-\mathrm{a}$ contradiction. Therefore $k \neq 2$ and so $k \geqq 3$. This completes the proof of statement (1) and with it the proof of the proposition.

Let $\mathfrak{B}$ be a variety of groups and suppose that $\mathfrak{B} \geqq \mathfrak{D}_{p}^{*}$. Let $\mathfrak{B}$ be an arbitrary minimal $p$-irregular subvariety of $\mathfrak{M}$. By Lemma 3.1 and the modular law

$$
\mathfrak{B} \leqq \mathfrak{D}_{p}^{*} \vee\left(\mathfrak{B} \wedge \mathfrak{B}_{p}^{\prime}\right)
$$

If $\mathfrak{B} \wedge \mathfrak{B}_{p}^{\prime}$ has class at most $3 p-3$, then so does $\mathfrak{B}$ and the previous proposition implies that $\mathfrak{B}=\mathfrak{D}_{p}^{*}$. This leads to the following theorem.

TheOREM 3.6. Let $\mathfrak{U}$ be an irregular variety of p-groups. Then $\mathfrak{D}_{p}^{*}$ is the only minimal p-irregular subvariety of $\mathfrak{U}$ if one or more of the following conditions are satisfied:

i) $p=2,3$ or 5 ,

ii) $\mathfrak{U} \leqq \mathfrak{N}_{3 p-3}$,

iii) $\mathfrak{U} \leqq \mathfrak{N}_{2} \mathfrak{A}$.

PROOF. In view of the comments preceding the statement of the theorem, it will suffice to show that, under any one of these conditions, a 2-generator group of exponent $p$ in $\mathfrak{U}$ has class at most $3 p-3$. Under condition ii) this is trivial and 
it follows from a theorem of Gupta [2] that under condition iii) any group of exponent $p$ in $\mathfrak{U}$ has class at most $2 p$.

If $p=2,3$ or 5 , then all finite 2-generator groups of exponent $p$ have class at most $3 p-3$. For $p=2$ or 3 , this is well known (see, for example, Huppert [4; III, 6]). For $p=5$, it follows from results of Kostrikin [5].

Finally, we use Theorem 3.6 to obtain criteria for regularity among varieties of $p$-groups.

THEOREM 3.7. Let $\mathfrak{U}$ be a variety of p-groups satisfying one or more of the conditions of Theorem 3.6. Then the following statements are equivalent:

a) $\mathfrak{U}$ is regular,

b) $\mathfrak{U}$ does not contain the wreath product of two cyclic groups of order $p$,

c) $\mathfrak{U}$ has the ( $p-1)^{\prime}$ th Engel congruence.

Proof. c) $\rightarrow$ b). This follows from the easily verified fact that the wreath product in question does not have the $(p-1)$ 'th Engel congruence.

b) $\rightarrow$ a). This follows from Theorem 3.6 and the definition of $\mathfrak{D}_{p}^{*}$.

a) $\rightarrow c$ c). Suppose that $\mathfrak{U}$ is regular. To show that $\mathfrak{U}$ has the $(p-1)$ 'th Engel congruence, it clearly suffices to suppose that $\mathfrak{U}$ has class $p$ and prove that $\mathfrak{U}$ then has the $(p-1)$ 'th Engel condition. Let $H=F_{2}(\mathfrak{H})$ and let $\{x, y\}$ be a free generating set of $H$. We must show that $[x,(p-1) y]=1$.

Since groups of exponent $p$ have the $(p-1)$ 'th Engel congruence, $H / \mathfrak{B}_{p}(H)$ has the $(p-1)$ 'th Engel condition. Thus $[x,(p-1) y] \in \mathfrak{B}_{p}(H)$. But $H$ is regular, and so each element of $\mathfrak{B}_{p}(H)$ is a product of $p$ 'th powers of commutators in $x$ and $y$. In particular,

$$
[x,(p-1) y]=\prod_{i} d_{i}^{p}
$$

By Lemma 1.2 of Weichsel [12], we can suppose that each $d_{i}$ has weight $p$ and so, by our Lemma 3.4, we can further suppose that each $d_{i}$ contains exactly one occurrence of $x$. But, in this case, each $d_{i}$ is either $[x,(p-1) y]$ or its inverse. Thus, for some integer $n,[x,(p-1) y]^{1+n p}=1$ and so $[x,(p-1) y]=1$, as $H$ is a $p$-group. This completes the proof.

\section{References}

[1] W. Brisley, 'On varieties of metabelian p-groups and their laws', J. Austral. Math. Soc. 7 (1967), 64-80.

[2] N. D. Gupta, 'Polynilpotent groups of prime exponent', Bull. Amer. Math. Soc. 74 (1968), 559-561.

[3] Graham Higman, 'Representations of general linear groups and varieties of groups', Proc. Internat. Conf. Theory of Groups, Austral. Nat. Univ. Canberra, August 1965, 167-183, (Gordon and Breach, New York, 1967). 
[4] B. Huppert, Endliche Gruppen I, (Springer, New York, 1967).

[5] A. I. Kostrikin, 'Solution of the restricted Burnside problem for exponent 5', Izv. Akad. Nauk SSSR. Ser. Mat. 19 (1955), 233-244 (Russian).

[6] A. I. Kostrikin, 'The Burnside problem', Izv. Akad. Nauk. SSSR Ser. Mat. 23 (1959), 3-34 (Russian) [English translation, Amer. Math. Soc. Transl. (2) 36 (1964), 63-99].

[7] L. G. Kovács and M. F. Newman, 'On critical groups', J. Austral. Math. Soc. 6 (1966), 237-250.

[8] L. G. Kovács and M. F. Newman, 'On non-Cross varieties of groups', J. Austral. Math. Soc. 12 (1971), 129-144.

[9] Avinoam Mann, 'Regular p-groups', Israel J. Math. 10 (1971), 471-477.

[10] H. Meier-Wunderli, 'Metabelsche Gruppen', Comment. Math. Helv. 25 (1951), 1-10.

[11] Hanna Neumann, Varieties of Groups (Springer, Berlin, 1967).

[12] Paul M. Weichsel, 'Regular p-groups and varieties', Math. Z. 95 (1967), 223-231.

[13] Paul M. Weichsel, 'Just irregular p-groups', Israel J. Math. 10 (1971), 359-363.

University of Manitoba

Winnipeg, Manitoba

Canada

Present address:

University of Melbourne

Parkville, Victoria 3052

Australia 